\title{
Can DRGs be over cooked? Or how fine a refinement is that?
}

\author{
C W Aisbett ${ }^{1,2}$ \\ From 26th Patient Classification Systems International (PCSI) Working Conference \\ Munich, Germany. 15-18 September 2010
}

\section{Introduction}

The thinking behind DRGs contains not only clinical concepts but also ideas drawn from health economics and statistics (particularly sampling theory). One example of the influence of economics is the literature on DRGs in reimbursement systems. DRGs are generally placed between fee for service and risk -adjusted capitation. An example of the influence of statistics is the assumption that within an appropriate group of hospitals, all hospitals will, on average, deliver the same resource content with the same quality of care to each DRG they treat.

Economics and statistics play a much wider role in case mix than illustrated by the examples above. For instance, the theory of incentives and the Generalized Central Limit Theorem (DRG swings and roundabouts) serve as a means of broadening hospital groups. This presentation, however, will focus on the economics and statistics to make its point.

To begin with, case-mix methods almost always involve a DRG classification system and ancillary information; for example, partitioning of same-day cases. Therefore, the placement of case mix on the continuum between fee for service and capitation is indicative of a range rather than a point. Some placements in the range may be more appropriate for an insurance-based system, while others more appropriate for a universal publicly funded health-care system.

Similarly, DRG systems vary in the number of case types they contain. Thus, smaller treatment populations may result in low-volume DRGs which do not provide sufficient central tendency for hospital comparison.

The contention of this paper is that an inpatient classification system suitable for the USA or Germany may not be appropriate for New South Wales (Australia) or

${ }^{1}$ LaetaPty Ltd, Randwick, New South Wales, Australia

Full list of author information is available at the end of the article
Ireland. The contribution of this paper is an assessment of the sensitivity of desired outcomes of patient classification to the assumptions underpinning case mix.

\section{Methods}

Analysis of some aspects of the hypothesis can be based on grouped data from NSW and Ireland; in particular, the effect of partitioning DRGs on admission type and day-case status. We test adjacent DRGs that are split between complex and other episodes for consistency of complex proportions across hospitals. The confinement DRGs (along with others) are considered.

Statistical analysis is also applied to the population assumption, both in overall size and in demographic consistency, across hospitals within the system(s). In particular, eye hospitals, women's hospitals and paediatric hospitals are assessed for the persistence of systemwide DRG cost and stay relativities.

A number of the other aspects (particularly those about incentives) must be argued on more a theoretical basis. This will include reference to the rich literature that exists.

\section{Results}

The maintenance of DRG cost and LOS relativities across all hospitals in a group is more difficult when the number of DRGs is low, but the evaluation of those relativities is more difficult when the number of DRGs is high. The trade-off is very clearly driven by population size.

The maintenance of DRG-derived incentives is reduced by increasing the number of case types, both through greater potential for over servicing and less reliable costs and relativities. Having fewer case types places greater emphasis on the treatment population assumption that underlies case mix, but improves the 
consistency of cost relativities and hence leads to fairer evaluation and funding.

\section{Conclusions}

DRGs can be over cooked, but it depends on the application. There is a fairly wide range of detail (e.g., number of case types) that may have little effect on the utility of the classification, and as a result many DRG refinements make little difference. However, any choice of classification should consider the nature of the treatment population and the health system.

There is a real danger that a large classification will be over-specified (impairing incentives) and inaccurately estimated when applied to a smaller treatment population in a public-health system.

\section{Author details}

'LaetaPty Ltd, Randwick, New South Wales, Australia. ${ }^{2}$ Department of Health and Children, Dublin, Ireland.

Published: 6 October 2010

doi:10.1186/1472-6963-10-S2-A24

Cite this article as: Aisbett: Can DRGs be over cooked? Or how fine a refinement is that?. BMC Health Services Research 2010 10(Suppl 2):A24.
Submit your next manuscript to BioMed Central and take full advantage of:

- Convenient online submission

- Thorough peer review

- No space constraints or color figure charges

- Immediate publication on acceptance

- Inclusion in PubMed, CAS, Scopus and Google Scholar

- Research which is freely available for redistribution

Submit your manuscript at www.biomedcentral.com/submit
C Biomed Central 